\title{
Digital Transformation in Medical Affairs Sparked by the Pandemic: Insights and Learnings from COVID-19 Era and Beyond
}

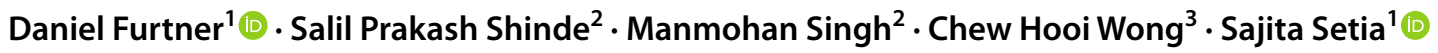

Accepted: 28 November 2021 / Published online: 31 December 2021

(c) The Author(s), under exclusive licence to Springer Nature Switzerland AG 2021

\begin{abstract}
A number of developments, including increasing regulatory and compliance scrutiny, increased transparency expectations, an increasingly vocal patient, patient centricity and greater requirements for real-world evidence, have driven the growth and importance of medical affairs as a trusted, science-driven partner over the past decade. The healthcare environment is shifting towards a digital, data-driven and payor-focused model. Likewise, medical affairs as a function within the pharmaceutical industry has become more "patient-centric" with strategic engagements embracing payers and patients apart from clinicians. The pandemic has impacted the healthcare industry as well as the function of medical affairs in numerous ways and has brought new challenges and demands to tackle. There is indeed a silver lining due to intense digital transformation within this crisis. The emerging digital innovation and new technologies in healthcare, medical education and virtual communications are likely to stay and advance further. In this review, we discuss how the digital transformation sparked by the pandemic has impacted the medical affairs function in pharmaceuticals and provide further insights and learnings from the COVID-19 era and beyond. Based on the learning and insights, digital innovation in three key strategic imperatives of medical affairs-HCP engagement, external partnerships and data generation will enable medical affairs to become futurefit as a strategic leadership function.
\end{abstract}

\section{Strategic and Intrinsic Value of the Medical Affairs Function in Pharmaceutical Organisations}

Medical affairs function has a broad scope of activities and responsibilities within the pharmaceutical industry, ranging from internal bridge between research and development (R\&D) and commercial functions, an external bridge between the organisation and external stakeholders, early access programs, investigator-initiated research, evidence generation (Phase IV real-world data, health economics

Daniel Furtner

daniel.furtner@transform-medcomms.com

1 Executive Office, Transform Medical Communications Limited, 184 Glasgow Street, Wanganui 4500, New Zealand

2 Regional Medical Affairs, Pfizer Corporation Hong Kong Limited, 21st Floor, Kerry Center, 683 King's Road, Quarry Bay, Hong Kong

3 Regional Medical Affairs, Pfizer Private Limited, 80 Pasir Panjang Road, \#16-81/82, Mapletree Business City, Singapore 117372, Singapore

\section{Key Points}

The challenges and changes posed by the COVID pandemic have accelerated a digital transformation in the pharmaceutical industry, which medical affairs function is well placed to lead and innovate.

The future of medical affairs is expected to be bright, and the function has been predicted to become one of the leading strategic pillars of the pharmaceutical enterprise.

To attain future fitness, medical affairs will have to demonstrate specific innovative digital capabilities in leading diverse partnerships and collaborations across the healthcare ecosystem. 
and outcome research), scientific publications, medical and public education, collaborations with patient organisations [1], to name a few.

Medical affairs is now empowered with a greater voice internally. In some organisations, it is now a key leadership function alongside commercial and R\&D [2]. The industry is shifting the focus of external interactions from sales/commercial to medical affairs due to health provider's preferences for non-promotional and scientific engagement by the industry [3]. A deep medical and scientific understanding of the disease is essential for the identification of potential impending modifications in better management of that disease, including pharmacologic treatment options [4]. Hence, the medical affairs role has shifted focus from being perceived as a supporting function to a strategic cross-functional partner adding value to the company and to the broader healthcare ecosystem [5].

Non-promotional initiatives of medical affairs colleagues with healthcare professionals (HCPs), academia and medical associations aim to add value for clinical practice and leverage subject matter expertise through various collaborations $[4,6-8]$. There is a growing representation by professional groups, networks and associations, which are advocating for the medical affairs' role and providing exchange platforms on international, regional and local levels (e.g., Medical Affairs Professional Society [MAPS]) [5, 9, 10]. These associations have also further defined and shaped the medical affairs profession, with MAPS in the lead, providing a fundamental elevator pitch in their white paper on the value of medical affairs as external-oriented scientific and medical leaders, representing the real-world clinical needs of healthcare professionals, patients, and other decision makers [11].

\section{Dynamic Evolving Role of Medical Affairs Function Before COVID-19 Pandemic}

One of the key roles of the medical affairs function is to bridge knowledge gaps among HCPs by enabling scientific exchange across various platforms (one-on-one, group discussions and medical education programmes) [12-14]. Medical affairs colleagues as scientific communicators are well-placed to help navigate data and insights, defining a clear evidence-based story for communication for HCPs, payers, patients, and internal stakeholders by applying a contextual narrative $[15,16]$.

In the last decade, the medical affairs function has been supporting pharmaceutical teams across the full product lifecycle and the associated customer journeys [2]. Medical affairs has also been engaging and collaborating with patients and patient organisations through strategic leadership, health communication, evidence generation and partnerships [17]. There has been a gradual shift from a disease-centred to a patient-centred strategy and from a product-led to a patient-led development process [18]. While generally no direct-to-patient advertising is allowed (except in USA, Hong Kong and New Zealand, with some restrictions) [1], patient advocacy groups play an important intermediary partner for medical and public affairs [19, 20]. Other endeavours, including studying and collecting patient-reported outcomes in clinical and especially realworld studies [21, 22], leading tailored disease awareness and patient education campaigns [23, 24], real-time diagnostic and treatment analytical support [25] have helped to not only empower patients but also generate valuable real-world data [26-29].

\section{Unprecedented Challenges for Medical Affairs During COVID-19 Pandemic}

The COVID-19 pandemic has significantly affected the healthcare sector and services at large, posing multiple constraints and challenges regarding the uncertainty about patients' timing and demand for services, the reality of multiple waves of resurgence, discontinuity in connection with patients, shift towards telecommunication and telemedicine, as well as disruption to the entire continuum of care [30]. There has been a significant impact of the pandemic on the pharmaceutical industry. The short-term impact included demand changes, regulation revisions, curtailment in research and development. Overall, there has been a slow-down in the growth, delays in non-COVID-19-relevant medicines approval, move towards self-sufficiency in production and supply chain at both global and local levels [31]. The indirect health footprint of the pandemic has had a far greater impact on chronic conditions [32]. People with chronic diseases (PWCD) not only encounter worse outcomes with COVID-19 infection but are also more adversely affected due to disruption in continuity and quality of care [32]. They experience panic, anxiety, stress, an increasingly sedentary lifestyle, and difficulty with drug supply, which all have affected adherence to medications and self-care for disease monitoring [33, 34]. However, individual self-care is dependent on health literacy, health knowledge as well as socioeconomic factors such as financial stresses [35]. The level of satisfaction related to online disease and self-care education during the pandemic was significantly lower in PWCD compared to people without chronic diseases [36].

The pandemic also impacted medical affairs function in numerous ways and brought new challenges and demands. The use of experimental medicines has been common during the pandemic (e.g., for repurposing of drugs 
or compassionate requests), but only medical affairs can respond to unsolicited off-label enquiries [37]. There has been reduced direct access to HCPs and institutions and an increase of virtual interactions and also a broader scope of engagement with HCPs, e.g., the impact of COVID-19 on non-related speciality practice and guidelines [38]. Significant changes in the patient-physician interaction have also shaped the patient-centricity priorities for medical affairs. Patients have increasingly been exploring convenient tools and guidance for self-management. Around $38 \%$ of clinicians indicated the need for virtual patient education tools in a recent survey by Medical Science Liaisons (MSLs) in Asia-Pacific [39].

There has been new unmet medical information and medical education needs [40]; for example, for tapering, missing, and discontinuing therapy (e.g., immunosuppressive medication), medical society recommendations on the management of patients during the pandemic for each speciality (especially for at-risk patients), guidance on switching from clinic-setting infusion therapy to home-based injections, COVID-19 vaccinations and concomitant medications, etc. Other newer requirements have included facilitating new study proposals to repurpose and investigate potential drug effects for COVID-19 patients, increased scope interactions with new stakeholders including national health institutions, infectious disease experts, support for vaccine launch and roll-out in companies that developed a COVID-19 vaccine, as well as managing challenges with patient enrolment, shift to digital methods for site selection and remote monitoring for the execution of real-world data generation [41].

There has also been some shift in medical affairs structure and resources: MSLs and field-based medical colleagues have reduced or stopped travelling with limited face-toface (F2F) interactions but are taking up more office-based responsibilities due to time saved through virtual interactions and limited access to HCPs [38, 42].

\subsection{Limited F2F Interactions for Medical Affairs with HCPs}

For many HCPs, the majority of contacts with medical affairs teams of the pharmaceutical industry were built up during F2F meetings pre-pandemic; thereby, informal networks, where expertise and ideas are being exchanged, were established. At a real-world meeting or conference, the chance of being personally confronted with important new developments is higher than by virtual attendance [42]. The need for social interactions has been called essential for physicians to develop or maintain personal relations facilitating creativity and networking [43]. The COVID pandemic has necessitated a shift from F2F to virtual meetings. Whilst benefits are seen with virtual interaction, such as the reduced cost and environmental impact due to travel, the larger reach, etc. [44], physicians experienced difficulties controlling their virtual agenda and struggled to contribute to the increasing activities of webinars, teaching courses, MSL calls and virtual collaboration opportunities within the industry [45].

The rapid shift to a virtual exchange format in the absence of clear regulations in place, has also led to fatigue and a feeling of being overwhelmed by the frequency [45]. Meanwhile, noteworthy best practice guidelines for virtual meetings have been published based on literature reviews, expert consultation and stakeholder surveys, and have laid out four phases of a virtual meeting cycle: Pre-planning considerations, Planning, Accomplishing conference goals through execution, gauging Response and Engaging the target audience for future cycles (PrePARE) [46]. As such, how well and to what extent virtual interactions will replace traditional F2F engagement seems to be a question of adaptation and implementation.

An MSL survey was recently conducted in the AsiaPacific region, using a literature review, an external survey of 210 HCPs, and an internal survey of 53 MSLs [41]. The survey identified the largest virtual competency gaps as (i) the inability to conduct highly interactive virtual engagement and (ii) ability to collect insights during virtual interactions. Thus, the medical affairs function is advised to close these gaps by providing holistic, individualised scientific engagements using advanced digital platforms and content transformation [41]. A recent HCP survey of a total of 511 HCPs (physicians, pharmacists, nurses, and physician assistants) has suggested that the pharmaceutical industry should further work on lack of awareness of medical information related to clinical practice guidelines, disease state, the safety of medicines, etc., and the speed at which responses are disseminated [47].

\section{Digital Fitness for Medical Affairs Function: Insights and Learnings for the Future}

The pandemic has necessitated the adoption of digital solutions in healthcare, such as telemedicine, artificial intelligence (AI), machine learning, connected healthcare devices, natural language processing, and virtual learning, which all lead to a digital healthcare system that is resilient to future challenges [48-51]. Agility to adapt has meant a fast shift in priorities towards COVID-related matters, a new understanding of customer and patient centricity and broader healthcare aspects associated with the pandemic, pioneering new technologies and leading customer interactions in a previously $\mathrm{F} 2 \mathrm{~F}$ and sales resource-orientated industry $[5,52]$. 


\subsection{Massive Open Online Courses (MOOCs) for Planning Virtual Medical Education}

Due to the COVID-19 pandemic, webinar usage by HCPs has increased by between 300 and $3250 \%$ in 2020 compared to 2019 [53, 54]. New ways of conducting medical education are likely to persist post-pandemic. Perception and perspectives for virtual Continuing Medical Education (CME) channels have transformed since the onset of the COVID-19 pandemic. While online platforms have emerged as the most common channels to earn CME credits, a recent survey also demonstrated that $60 \%$ of physicians now consider online/ virtual CME platforms comparable to in-person CME activities [55]. Also, $72 \%$ of physicians who didn't use online learning platforms for CME credits prior to COVID-19 now intend to increase usage of online learning platforms [56]. Another survey among 526 urology providers from 56 countries revealed networking opportunities were identified as high in a F2F meeting, yet online webinars were more costeffective, and learning opportunities and reach of the audience was higher for hybrid meetings. Post-COVID-19, the conference format of choice was a hybrid conference $(n=$ $199,51 \%)$ followed by a webinar-only format $(n=95,25 \%)$ [57]. Similar preferences were also reported from another survey of 191 HCPs from India, with $93 \%$ of respondents being open to innovative formats of learning [42].

In a cross-sectional, internet-based survey on physicians' attitude towards webinars and online education during the pandemic, among 326 physicians, the majority reported an initial satisfaction. Satisfaction was higher for "international conferences" and "teaching courses" versus pharmaceutically sponsored events $(74.5 \%$ vs $41.4 \%, p \leq 0.001)$. This notable disparity in satisfaction might be attributed to the aforementioned higher number, frequency, and overlapping activities of pharmaceutical-sponsored meetings, probable perceived biases, or dominance of early COVID-19-related topics at that time. Moreover, international conferences are annual events that usually lack commercial biases, and are eligible for higher CME accreditation, than industry-sponsored events [45]. However, this provides useful insights for medical affairs function in improving and planning online medical education activities during and after the pandemic.

With increased interest from physicians for online CMEs, medical affairs is expected to create more tailored virtual medical education content, align teams with launching specialised medical education programmes based on current practice needs and design online in-house platforms or rather out-source to third-party technological platforms that partner directly with CME faculty, which could also add value and credibility [56]. This can be achieved by adopting MOOCs-massive open online courses, a cost-effective way to deliver readily accessible content to health providers, meeting their flexible schedule. MOOCs refer to online learning where online courses are accessible at little or no cost, and can reach large numbers of learners. Additionally, for optimum results, the platform for MOOCs also needs the functionality for learners to raise questions, share and discuss different practices, issues, and local data, and to connect and network with fellow learners and others for research and other purposes. The case-study-based interactive multimedia MOOCs with integration into blended learning with live meetings will be the ideal option for most future CMEs versus webinars and virtual medical education used during COVID. As MOOC CMEs would involve accreditation and tracking, they need to be delivered via a platform that has an embedded learning management system (LMS) [58].

Defining educational objectives in partnership with independent organisations, aligning the content with evidencebased data, obtaining endorsement from accreditation sources, and using an independent partner to coordinate the delivery of the programme, can lead to credible industryfunded CME that provides value and leads to HCP satisfaction. Expert review of content by independent organisations or a CME-faculty would ensure that CME programmes are not used for commercial promotion [58]. Best practice examples of effective collaborations for MOOC already existed pre-pandemic where an independent CME-faculty partnered with industry to develop educational programmes, endorsing and hosting its content on a professional specialist association platform for its members.

An insight-led example from the Asia-Pacific region is ASPIRE- the Asia-Pacific Initiative for Rheumatology nurse Education [59]; an independent faculty-led initiative established as a response to the increasing challenges experienced by rheumatologists in managing their patient caseload and the recognition of the evidence-based practices demonstrating the value of the nurse specialists in clinical practice. The organisational and funding support for meetings and workshops were provided by the industry. Nurse educational needs, determined from surveys conducted in the region, were identified to craft a curriculum that could provide nurses with the knowledge and skills that extended beyond their initial education and training. Working with national rheumatology associations and nursing bodies across the Asia-Pacific region, efforts have been strategically focused on expanding the reach of ASPIRE by the dissemination and implementation of existing modules. To facilitate this, core modules along with other resources-including tutorial videos for disease assessments, pre-and post-meeting knowledge tests- have been endorsed by the Education Committee of APLAR - the Asia Pacific League of Associations for Rheumatology - and published on its website within an Implementation Toolkit to ensure a consistent training structure (http://www.aplar.org/education_page/aspire/) [59]. To measure the success of ASPIRE workshop trainings on a participant level of knowledge, confidence, attitudes and 
beliefs using a before-after-control-impact survey was carried out, demonstrating a significant increase in knowledge and confidence [60].

\subsection{Digital Storytelling}

The scope and delivery methodology for scientific or educational storytelling to HCPs and public had already been advancing long before the pandemic through an evolving communication strategy, adopting elements like scientific descriptions [61], addressing knowledge deficits through reflection [61, 62], narrative medicine for real-world case studies, etc. [63]. Since the pandemic, traditional medical publications have increasingly experienced new life by embracing digital innovation, with publication enhancements such as animated figures, e-posters, and author videos. Digital transformations in medical and health education storytelling have enabled the visualisation of presentation in captivating infographic formats and animated illustrations make data even more interesting and also enhance shared decision making $[64,65]$. Digital storytelling enables knowledge sharing and critical thinking for available evidence with patients, caregivers, HPCs and policy makers, enabling them to be more engaged while making a health decision $[65,66]$.

Plain language summaries (PLS) are short summaries of medical publications but written in every-day language that can be easier understood by patients and caregivers [67]. Increasingly, PLS are becoming an established part of company-sponsored research publications [68-70] and recently, Open Pharm, a multi-sponsor collaboration of publishers, pharmaceutical companies, patients, academics, non-pharmaceutical funders and regulators, have provided a set of recommendations for PLS, that should also be able to be indexed in medical search engines such as PubMed [67]. Prioritising the generation of PLS and other digital storytelling tools by medical affairs teams is expected to continue post-pandemic.

\subsection{Execution of Successful Digital Transformations During and Post-pandemic}

Medical affairs has built digital engagement through the accelerated adoption of digital solutions by field leadership teams during the pandemic, as well as the automation of medical information solutions [71]. Yet, digital technology is not restricted to using digital channels for scientific communication. It includes the use of AI, analysing big data when available, incorporating data analytics in insights, exploring digital models of access and adopting a digital mindset. This would mean that medical affairs colleagues would have to adapt to modern technology and incorporate a digital component for all professional activities [38].
During the pandemic, there are also examples for successful real-world data generation related to clinical practice educational gaps and perspectives of HCPs using digital tools like SurveyMonkey, which can create anonymised surveys [72-75]. These also stand as examples for leading data generation projects by MSLs through virtual interactions with HCPs [76].

The digital transformation in medical affairs requires the provision of infrastructure plus training to acquire capabilities and digital literacy as well as changes in management and support by senior leadership, which will empower medical affairs to drive it. On an individual company level, this means a competitive disadvantage if not acted upon. It also requires a shift in the strategic medical imperatives: big data, real-world evidence, AI, and partnerships in patient-centred ecosystems need to play a role in medical affairs' strategy as well as the definition of success factors, key performance indicators and metrics [52].

Metrics for digital transformation can be quantitative and qualitative and should be measured in defined intervals. Some examples of quantitative metrics could be: number of software users relative to the number of licenses purchased, number of processes performed, productivity indicators [77]. Examples of qualitative metrics could relate to skills and knowledge acquired (digital literacy), trust, satisfaction, attitudes and behaviours [78]. Artificial intelligence can be used to accelerate and synthesise insights from real-world data. AI can also be used to optimise digital channels for medical content and to understand the patient experience [52]. Analysing the behaviour and expectations of patients and physicians following trends are enabled by technology advances, e.g., usage of mobile devices, remote data access, availability of real-time information, acceptance of social media as a new medium for interaction [79]. As an example, digital HCPs who have a large reach via digital platforms are augmenting the ranks of 'traditional' HCPs whose academic papers and presentations dominate leading congresses and journals. Medical affairs will need to engage with HCPs who blend the strengths of both traditional and digital leaders [80].

Figure 1 describes both pre-COVID and post-COVID evolution of medical affairs function.

\section{Outlook: How Could Medical Affairs Look in the Next 10 Years?}

Undoubtedly, the COVID pandemic has accelerated the digital transformation and put medical affairs at the forefront. Medical affairs may transition further into a strategic leadership role due to its professional standing, scientific knowledge, and responsibilities connecting internal functions. Medical affairs has been predicted to become the third strategic pillar of the pharmaceutical enterprise alongside 


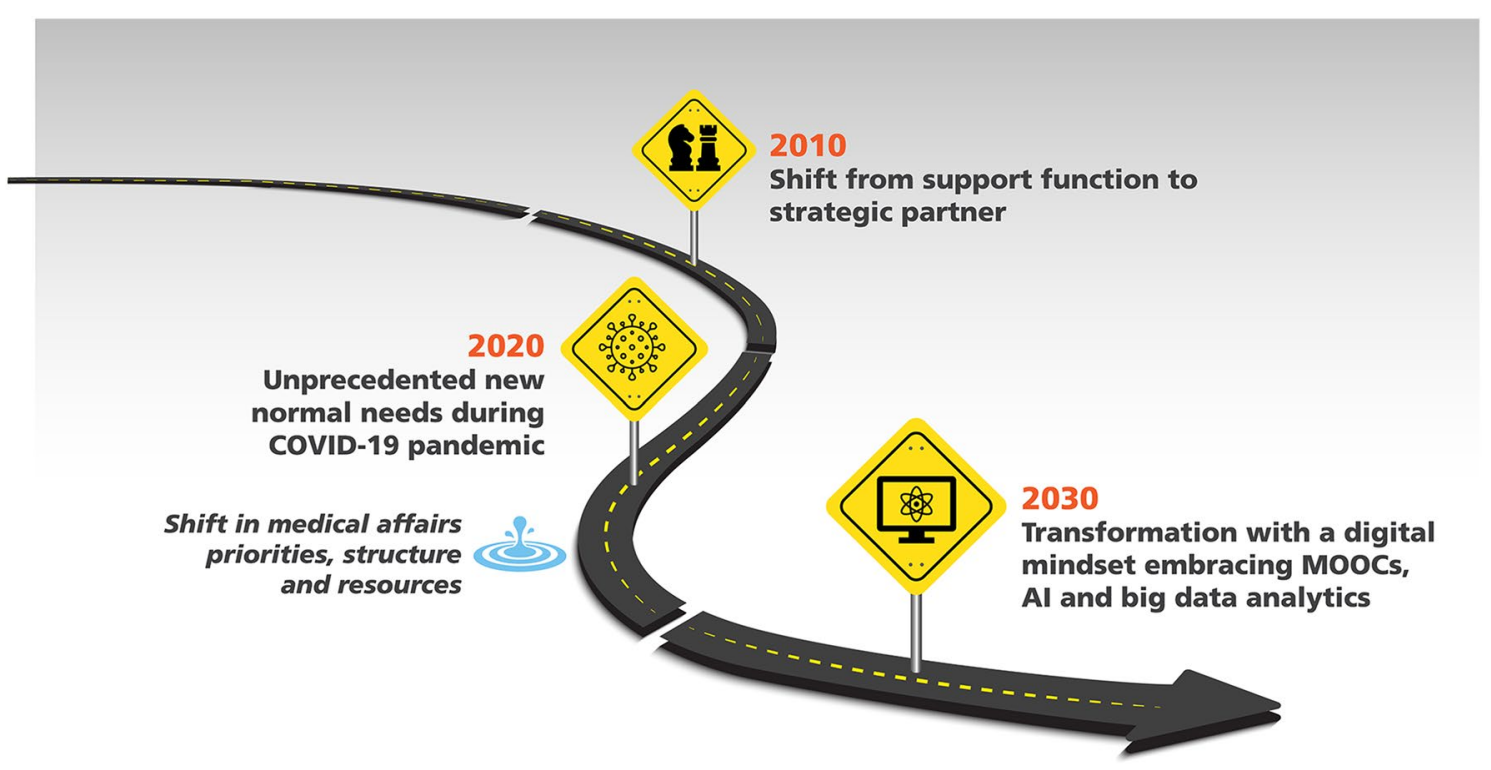

Fig. 1 From support to digital leadership-evolution of medical affairs function in pharma. The medical affairs function has experienced a shift in priorities, structure and resources due to the COVID pandemic. Some of these transformations are here to stay with a digi-

its $\mathrm{R} \& \mathrm{D}$ and commercial functions due to the accelerated volume of scientific data generated and the complexities in the navigation and positioning of science to inform interactions with stakeholders and ultimately improve patient outcomes [81]. The digital fitness for medical affairs would still continue to require close alignment with commercial, market access and public affairs departments. However, the extent of such internal collaboration would depend on the stringency of internal and external regulations.

Post-pandemic, medical affairs key strategic imperatives can be segregated into three broad areas-HCP engagement, external partnerships, data generation. These are also briefly illustrated in Fig. 2.

\subsection{HCP Engagement}

Medical affairs, and especially its in-field teams, will engage a broader set of healthcare stakeholders, including digital versatile and influential HCPs, payers, and patient advocacy groups. Engagement will need to be high quality and tailored to individual needs and preferences. Digital tools will replace many of today's F2F interactions, informed by analytics and data [81]. The shift to a digital transformation-sparked by the COVID pandemic_-will also see medical affairs adopting an omnichannel customer approach, contributing to the integration of digital touchpoints that will put HCPs and patients versus the drug at the core. The tal mindset embracing MOOCs, AI and big data analytics. By 2030, medical affairs will also need to lead digital data generation to be established as a patient-centric scientific partner of choice. AI artificial intelligence, MOOCs Massive Open Online Courses

key here will be that medial affairs delivers a data-driven, customer-centric experience that flows seamlessly across the different channels and multiple devices. Capturing and analysing data in one centralised location with corresponding business processes and robust governance will be key as well as keeping consistency across channels [82].

\subsection{External Partnerships}

External partnerships are essential to continued innovation, spanning across industries, academia, non-profit and public sectors and across the full spectrum of R\&D and commercial activities [83]. External partnerships, including private-public partnerships (PPPs) with academic institutions, research consortia, patient advocacy groups, realworld registries/databases, health technology assessment (HTA) bodies, payers, technology and analytics vendors, will grow-mostly led by medical affairs [84]. Strategic alliances will be designed to share risk and costs, especially for data generation but also the total cost of care and to improve patient outcomes. Formal partnerships between medical affairs and payer decision-makers may aim to accomplish this via managed entry agreements or outcomes-based contracting [81]. The external partnerships may also involve other pharmaceutical companies, as identified, and agreed upon by senior management to ensure credible and impactful value to external stakeholders [10]. 




Fig. 2 Key strategic imperatives for medical affairs in next 10 years. Innovation in three key strategic imperatives of medical affairs- $-\mathrm{HCP}$ engagement, external partnerships and data generation will enable medical affairs to become future-fit as a strategic leadership function. Organisational readiness in these strategic imperatives is, however,

\subsection{Data Generation}

Meaningful data generation, especially through the means of digitalisation, will be at the core of medical affairs future responsibilities and will stretch from clinical studies to real-world data. Data will be more specific to answer key research questions, will be repurposed-for instance, post hoc Phase 2 and 3 clinical trial sub-analyses or real-world evidence (RWE) data for regulatory use - and will integrate options of real-time measurements and availability. Patientcentricity in data generation will underline the shift from mere clinical, efficacy-and-safety-based, functional and survival driven research endpoints towards humanistic and patient-reported outcomes [81].

Real-world evidence complements the evidence generated by randomised-controlled trials (RCTs) through data in real-life patient settings. Established RWE, such as postmarketing safety surveillance or post-approval commitment studies, will continue to evolve from an effectiveness perspective in addition to the traditional collection of safety necessary to ensure the strategic value of medical affairs that could provide optimum value in terms of leadership in innovation and technology for this function to be regarded as the scientific partner of choice. $H C P$ healthcare professional

information. RWE will continue to be elevated to satisfy reimbursement and for regulatory purposes and will benefit from larger acceptance from such bodies [85].

Clinical decision-support systems are rapidly enhancing healthcare decision making using AI medical domains [86]. Research laboratories and technology firms are developing approaches using AI or big data for screening, diagnoses and for predicting response to treatments with equal or greater accuracy than human clinicians [87]. Cutting edge AI models in conjunction with high-quality, real-world data are expected to lead to improved prognostic and diagnostic models [88]. There will be exponential growth of available data. "Big data" will be utilised to advance prediction, performance, discovery, and comparative effectiveness research to address the complexity of patients, populations, and organisations [89]. Exploiting new tools and technologies to extract meaning from large-volume information has the potential to drive future change in clinical practice, from personalised therapy to population screening and electronic health record mining [90]. 


\section{Summary and Conclusions}

The challenges and changes posed by the COVID pandemic have accelerated a digital transformation in the pharmaceutical industry, which medical affairs is well placed to lead and expand. The future of medical affairs is expected to be bright, and the function has been predicted to become the third strategic pillar of the pharmaceutical enterprise alongside its R\&D and commercial functions. To attain future fitness, medical affairs will have to leverage technological advances and demonstrate digital competency and mindset change, extend partnerships and collaborations across the healthcare ecosystem, acquire business leadership acumen, knowledge acquisition and self-development, and the ability to generate real-world evidence with data science, AI technology and analytics. It also includes widening the scope of partnerships with technology providers and HCP digital leaders or even other pharmaceutical companies.

However, organisational readiness is required for adequate resources in innovation and technology to ensure impactful collaboration with decision makers and external stakeholders.

\section{Declarations}

Funding The research and preparation for the manuscript received no fees, grant or sponsorship from any funding agency in the commercial, public, or not-for-profit sectors.

Conflict of interest All the authors (Dr Daniel Furtner, Dr Salil Shinde, Dr Manmohan Singh, Dr Chew Hooi Wong and Dr Sajita Setia), report no conflicts of interest in this work. The views and opinions expressed in the article are those of the authors and do not represent or reflect in any way the official policy or position of their current or previous employers.

Availability of data and material Not applicable.

Ethics approval Not applicable.

Consent Not applicable.

Author contributions All the authors (DF, SS, MS, CHW and SS) participated in the conception of the idea, design and interpretation of the facts and data. DF and SS participated in manuscript writing and all authors were engaged in revising it for scientific content and approval before its submission for publication. All the authors have also read and approved the final version.

Code availability Not applicable.

\section{References}

1. Setia S, Ryan NJ, Nair PS, et al. Evolving role of pharmaceutical physicians in medical evidence and education. Adv Med Educ Pract. 2018;9:777-90.
2. Lush L. Fishawack. What does the future hold for Medical Affairs? https://fishawack.com/news/what-does-the-future-hold-for-medic al-affairs/. Accessed 20 Sep 2021.

3. Jandhyala R. Personal selling and its effectiveness in generating sales: an assessment of: 'promotional', 'non-promotional' personal selling and 'non-personal' selling as part of the Promotional Mix in the Pharmaceutical Industry 2015.

4. Sweiti H, Wiegand F, Bug C, et al. Physicians in the pharmaceutical industry: their roles, motivations, and perspectives. Drug Discovery Today. 2019;24(9):1865-70.

5. Bedenkov A, Rajadhyaksha V, Beekman M, et al. Developing medical affairs leaders who create the future. Pharm Med. 2020;34(5):301-7.

6. Moss RJ, Smith EB, Anderson G, et al. A survey of key opinion leaders to support curriculum development in advanced medical science liaison training. Ther Innov Regul Sci. 2015;49(1):45-9.

7. Patil S. Early access programs: benefits, challenges, and key considerations for successful implementation. Perspect Clin Res. 2016;7(1):4-8.

8. Tham TY, Tran TL, Prueksaritanond S, et al. Integrated health care systems in Asia: an urgent necessity. Clin Interv Aging. 2018;13:2527-38.

9. Theron P, Britland M, Holder D, et al. Promoting best practices for medical science liaisons position statement from the APPA, IFAPP, MAPS and MSLS. Ther Innov Regul Sci. 2021;55(6):1139-44.

10. Bedenkov A, Rajadhyaksha V, Moreno C, et al. The 7+ habits of highly effective medical directors. Pharm Med. 2021;35(5):267-79.

11. Kremer CM, Piliero PJ, Plessis Dd. Medical Affairs Professional Society. Communicating the value of medical affairs: A MAPS white paper. https://medicalaffairs.org/wp-content/uploads/2020/ 08/MAPS-White-Paper_Comm-Value-of-Medical-Affairs_May20 201.pdf. Accessed 21 Sep 2021.

12. Peng J, Rushton M, Johnson C, et al. An international survey of healthcare providers' knowledge of cardiac complications of cancer treatments. Cardiooncology. 2019;5:12-12.

13. Leonard E, Wascovich M, Oskouei S, et al. Factors affecting health care provider knowledge and acceptance of biosimilar medicines: a systematic review. J Manag Care Spec Pharm. 2019;25(1):102-12.

14. Kristensen N, Nymann C, Konradsen H. Implementing research results in clinical practice- the experiences of healthcare professionals. BMC Health Serv Res. 2016;16:48-48.

15. Sundin A. Stockholm Environment Institute. Make your science sticky-storytelling as a science communication tool. https:// www.sei.org/perspectives/make-science-sticky-storytelling-scien ce-communication-tool/. Accessed 22 Sep 2021.

16. Dohan D, Garrett SB, Rendle KA, et al. The importance of integrating narrative into health care decision making. Health Affairs (Project Hope). 2016;35(4):720-5.

17. Ashkenazy R. Building the case for developing a medical affairs patient-centric framework collaboratively. Drug Discovery Today. 2020;25(3):475-9.

18. du Plessis D, Sake JK, Halling K, et al. Patient centricity and pharmaceutical companies: is it feasible? Ther Innov Regul Sci. 2017;51(4):460-7.

19. Rose DM, Marshall R, Surber MW. Pharmaceutical industry, academia and patient advocacy organizations: what is the recipe for synergic (win-win-win) collaborations? Respirology (Carlton, Vic). 2015;20(2):185-91.

20. Roennow A, Sauvé M, Welling J, et al. Collaboration between patient organisations and a clinical research sponsor in a rare disease condition: learnings from a community advisory board and best practice for future collaborations. BMJ Open. 2020;10(12):e039473. 
21. Stergiopoulos S, Michaels DL, Kunz BL, et al. Measuring the impact of patient engagement and patient centricity in clinical research and development. Ther Innov Regul Sci. 2020;54(1):103-16.

22. Michaels DL, Lamberti MJ, Peña Y, et al. Assessing biopharmaceutical company experience with patient-centric initiatives. Clin Ther. 2019;41(8):1427-38.

23. Bartlett S, Dezii C, Sandt K. The universal patient language: a set of resources and tools to design patient communications that support better health literacy. Stud Health Technol Inform. 2020;269:264-74.

24. Camacho N. Patient empowerment: consequences for pharmaceutical marketing and for the patient-physician relationship. In: Innovation and marketing in the pharmaceutical industry: Emerging practices, research, and policies. New York: Springer; 2014. p. 425-55.

25. Debong F, Mayer H, Kober J. Real-world assessments of mySugr Mobile Health App. Diabetes Technol Therap. 2019;21(S2):S2-35-S32-40.

26. Fendrick AM, Brixner D, Rubin DT et al. Sustained long-term benefits of patient support program participation in immunemediated diseases: improved medication-taking behavior and lower risk of a hospital visit. J Managed Care Specialty Pharmacy. 2021;27:1086-1095.

27. Ganguli A, Clewell J, Shillington AC. The impact of patient support programs on adherence, clinical, humanistic, and economic patient outcomes: a targeted systematic review. Patient Prefer Adherence. 2016;10:711-25.

28. Van den Bosch F, Ostor AJK, Wassenberg S, et al. Impact of participation in the adalimumab (Humira) patient support program on rheumatoid arthritis treatment course: results from the PASSION study. Rheumatol Ther. 2017;4(1):85-96.

29. Brixner D, Rubin DT, Mease P, et al. Patient support program increased medication adherence with lower total health care costs despite increased drug spending. J Manag Care Spec Pharm. 2019;25(7):770-9.

30. Malani R, Revenig L, Santo T, et al. McKinsey \& Company. Preparing for the next normal now: How health systems can adopt a growth transformation in the COVID-19 world. https:// www.mckinsey.com/industries/healthcare-systems-and-servi ces/our-insights/preparing-for-the-next-normal-now-howhealth-systems-can-adopt-a-growth-transformation-in-thecovid-19-world. Accessed 20 Aug 2021.

31. Ayati N, Saiyarsarai P, Nikfar S. Short and long term impacts of COVID-19 on the pharmaceutical sector. Daru J Faculty Pharm Tehran Univ Med Sci. 2020;28(2):799-805.

32. Danhieux K, Buffel V, Pairon A, et al. The impact of COVID19 on chronic care according to providers: a qualitative study among primary care practices in Belgium. BMC Fam Pract. 2020;21(1):255.

33. Singh K, Kaushik A, Johnson L, et al. Patient experiences and perceptions of chronic disease care during the COVID19 pandemic in India: a qualitative study. BMJ Open. 2021;11(6):e048926.

34. Gupta SK, Lakshmi PVM, Kaur M, et al. Role of self-care in COVID-19 pandemic for people living with comorbidities of diabetes and hypertension. J Family Med Prim Care. 2020;9(11):5495-501.

35. Duggan M. Victoria University, Mitchell Institute. Self-care: a game changer for everyone's health. https://www.vu.edu.au/ mitchell-institute/health/self-care-a-game-changer-for-everyoneshealth. Accessed 20 Sep 2021

36. Kor PPK, Leung AYM, Parial LL, et al. Are people with chronic diseases satisfied with the online health information related to COVID-19 during the pandemic? J Nurs Scholarship. 2021;53(1):75-86.
37. Ojo-Aromokudu O. The Pharmaceutical Journal. The pandemic's challenge to evidence-based medicine shows off-label use is an important tool. https://pharmaceutical-journal.com/article/letters/ the-pandemics-challenge-to-evidence-based-medicine-shows-offlabel-use-is-an-important-tool. Accessed 20 Sep 2021.

38. Rajadhyaksha VD. Medical affairs post-COVID 19: are we ready to take the baton? Perspect Clin Res. 2020;11(3):124-7.

39. Mah KW, Lo K, Elegant V. Medical Affairs Professional Society. A Year with COVID-19: Scientific Engagements Pulse Check. https://medicalaffairs.org/covid-19-scientific-engagements-pulsecheck/. Accessed 14 Nov 2021.

40. Jarvis S. MJH Life Sciences ${ }^{\mathrm{TM}}$ and Pharmaceutical Executive. How Medical Affairs Teams Need to Adapt to COVID-19. https:// www.pharmexec.com/view/how-medical-affairs-teams-needadapt-covid-19. Accessed 20 Sep 2021.

41. Mah KW, Lo K, Elegant V. Medical Affairs Professional Society. Reimagining Scientific Engagements During COVID-19. https:// medicalaffairs.org/reimagining-scientific-engagements-duringcovid-19/. Accessed 13 Nov 2021.

42. Ghosh R, Mohanasundaram S, Shetty S, et al. Preparing for the next normal: transformation in the role of medical affairs following the COVID-19 pandemic. Pharm Med. 2021:1-6.

43. Hilbe W, Pircher A, Füreder T, et al. Creating the "new normal" post-Coronavirus world-web-communication replacing face-toface interaction. Memo. 2020;13(3):245-6.

44. Kruger JM, Chowers I. The ethical advantages of video conferencing in medical education. Med Educ Online. 2020;25(1):1787310-1787310.

45. Ismail II, Abdelkarim A, Al-Hashel JY. Physicians' attitude towards webinars and online education amid COVID-19 pandemic: when less is more. PLoS ONE. 2021;16(4):e0250241.

46. Rubinger L, Gazendam A, Ekhtiari S, et al. Maximizing virtual meetings and conferences: a review of best practices. Int Orthop. 2020;44(8):1461-6.

47. Hermes-DeSantis ER, Hunter RT, Welch J, et al. Preferences for accessing medical information in the digital age: health care professional survey. J Med Internet Res. 2021;23(6):e25868.

48. Farrugia G, Plutowski RW. Innovation lessons from the COVID19 pandemic. Mayo Clin Proc. 2020;95(8):1574-7.

49. Woolliscroft JO. Innovation in Response to the COVID-19 Pandemic Crisis. Acad Med. 2020;95(8):1140-2.

50. Mirzaian E, Franson KL. Leading a digital transformation in pharmacy education with a pandemic as the accelerant. Pharmacy (Basel). 2021;9(1):19.

51. Jumreornvong $\mathrm{O}$, Yang $\mathrm{E}$, Race $\mathrm{J}$, et al. Telemedicine and medical education in the age of COVID-19. Acad Med. 2020;95(12):1838-43.

52. Bedenkov A, Moreno C, Agustin L, et al. Customer centricity in medical affairs needs human-centric artificial intelligence. Pharm Med. 2021;35(1):21-9.

53. Nepal PR. Role of webinars in medical educations during pandemic of COVID 19. Eastern Green Neurosurgery. 2020;2(2):1-2.

54. Navia A, Berner JE, Pereira N, et al. Have we passed the peak? The COVID-19 plastic surgery webinar pandemic. Aesthetic Surg J. 2020;40(9):NP569.

55. Wayne DB, Green M, Neilson EG. Medical education in the time of COVID-19. Sci Adv. 2020;6(31):eabc7110.

56. Ernst \& Young LLP. Impact of COVID-19 on physician interactions and learning: Survey finding. 2020. https://assets.ey.com/ content/dam/ey-sites/ey-com/en_us/topics/life-sciences/ey-hcpcovid-survey-findings.pdf. Accessed 20 Sep 2021.

57. Hameed BZ, Tanidir Y, Naik N, et al. Will "Hybrid" meetings replace face-to-face meetings post COVID-19 era? Perceptions and Views From The Urological Community. Urology. 2021;156:52-57. 
58. Setia S, Tay JC, Chia YC, et al. Massive open online courses (MOOCs) for continuing medical education - why and how? Adv Med Educ Pract. 2019;10:805-12.

59. Oliver S, Tam LS, Suet-Kei Kwok G, et al. The Asia-Pacific Initiative for Rheumatology Nurse Education: current gaps, programme development and future outlook. Musculoskeletal Care. 2020;18(3):397-403.

60. Oliver S, Bradbury L, Mu R, et al. Asia-Pacific initiative for rheumatology nurse education: an impact survey in China. J Nurs Educ Pract. 2020;11:8-18.

61. Liao H-C, Wang Y-H. Storytelling in medical education: narrative medicine as a resource for interdisciplinary collaboration. Int $\mathrm{J}$ Environ Res Public Health. 2020;17(4):1135.

62. Lutz G, Roling G, Berger B, et al. Reflective practice and its role in facilitating creative responses to dilemmas within clinical communication-a qualitative analysis. BMC Med Educ. 2016;16(1):301.

63. Camden VJ, Molloy EJ, Bearer CF. Our new feature: narrative medicine. Pediatr Res. 2020;88(3):343-4.

64. Krendyukov A, Nasy D. Evolving communication with healthcare professionals in the pharmaceutical space: current trends and future perspectives. Pharm Med. 2020;34(4):247-56.

65. Park E, Forhan M, Jones CA. The use of digital storytelling of patients' stories as an approach to translating knowledge: a scoping review. Res Involv Engagem. 2021;7(1):58-58.

66. Zarei A, Mojtahedzadeh R, Mohammadi A, et al. Applying digital storytelling in the medical oncology curriculum: effects on students' achievement and critical thinking. Ann Med Surg (2012). 2021;70:102528.

67. Rosenberg A, Baróniková S, Feighery L, et al. Open Pharma recommendations for plain language summaries of peerreviewed medical journal publications. Curr Med Res Opin. 2021;37(11):2015-6.

68. Lobban D, Gardner J, Matheis R. Plain language summaries of publications of company-sponsored medical research: what key questions do we need to address? Curr Med Res Opinion. 2021. https://doi.org/10.1080/03007995.2021.1997221.

69. Smith R. Improving and spreading plain language summaries of peer-reviewed medical journal publications. Curr Med Res Opin. 2021;37(11):2017-8.

70. King K, Yelling S, Stones S, et al. The Role of Plain-Language Summaries in Communicating Clinical Trial Data. https://medic alaffairs.org/plain-language-summaries-clinical-trial/ Accessed 20 Sep 2021.

71. Dwyer MA, Lal S. European Pharmaceutical Review. Digital agility and customer-centricity-imperatives for Medical Affairs in a post-COVID world. https://www.europeanpharmaceuticalrev iew.com/article/133844/digital-agility-and-customer-centricityimperatives-for-medical-affairs-in-a-post-covid-world/. Accessed 20 Sep 2021.

72. Morrison EH, Michtich K, Hersh CM. How the COVID-19 pandemic has changed multiple sclerosis clinical practice: results of a nationwide provider survey. Multiple Sclerosis Related Disord. 2021;51:102913

73. Robbins JB, England E, Patel MD, et al. COVID-19 impact on well-being and education in radiology residencies: a survey of the association of program directors in radiology. Acad Radiol. 2020;27(8):1162-72.

74. Vilovic T, Bozic J, Vilovic M, et al. Family physicians' standpoint and mental health assessment in the light of COVID-19 pandemic-a nationwide survey study. Int J Environ Res Public Health. 2021;18(4):2093.

75. Kidd VD, Vanderlinden S, Hooker RS. A National Survey of postgraduate physician assistant fellowship and residency programs. BMC Med Educ. 2021;21(1):212-212.

76. Singh M, Jain A, Fang W, et al. Educational needs, perception, and perspectives of oncologists regarding compassionate use programs in Asia. Curr Med Res Opinion. 2021;37:1-7.

77. WalkMe. How do you measure digital transformation? 5 metrics to know. https://blog.walkme.com/measure-digital-transforma tion/. Accessed 20 Sep 2021.

78. Organisation for Economic Co-operation and Development (OCED). Measuring the Digital Transformation: A Roadmap for the Future (Excerpt). https://www.oecd.org/going-digital/measu rement-roadmap.pdf. Accessed 20 Sep 2021.

79. Evers M, Fleming E, Ghatak A, et al. Pharma medical affairs: 2020 and beyond. New York: McKinsey \& Company; 2014.

80. Pharmaspectra. To maximize KOL impact, Medical Affairs needs a digital strategy too. https://www.pharmaspectra.com/resources/ features/to-maximize-kol-impact-medical-affairs-needs-a-digitalstrategy-too/. Accessed 20 Sep 2021.

81. Evers M, Ghatak A, Suresh B, et al. A vision for medical affairs in 2025. McKinsey \& Co. 2019.

82. Patel M. Medical Affairs: Meeting the Challenges of COVID19 and Omnichannel Engagement. https://www.veeva.com/blog/ medical-affairs-meeting-the-challenges-of-covid-19-and-omnic hannel-engagement/. Accessed 22 Sep 2021.

83. Feczko JM. The role of knowledge markets in reducing drug development costs and improving clinical care. https://www.oecd. org/health/biotech/41595326.pdf. Accessed 21 Sep 2021.

84. de Vrueh RLA, Crommelin DJA. Reflections on the future of pharmaceutical public-private partnerships: from input to impact. Pharm Res. 2017;34(10):1985-99.

85. Khosla S, White R, Medina J, et al. Real world evidence (RWE)-a disruptive innovation or the quiet evolution of medical evidence generation? F1000Research. 2018;7:111.

86. Montani S, Striani M. Artificial intelligence in clinical decision support: a focused literature survey. Yearb Med Inform. 2019;28(1):120-7.

87. Davenport $\mathrm{T}$, Kalakota $\mathrm{R}$. The potential for artificial intelligence in healthcare. Future Healthc J. 2019;6(2):94-8.

88. Magrabi F, Ammenwerth E, McNair JB, et al. Artificial intelligence in clinical decision support: challenges for evaluating AI and practical implications. Yearb Med Inform. 2019;28(1):128-34.

89. Krumholz HM. Big data and new knowledge in medicine: the thinking, training, and tools needed for a learning health system. Health affairs (Project Hope). 2014;33(7):1163-70.

90. Hulsen T, Jamuar SS, Moody AR, et al. From big data to precision medicine. Front Med (Lausanne). 2019;6:34-34. 